\title{
Linearly Polarized 64-element Antenna Array for mm-Wave Mobile Backhaul Application
}

\author{
Marko Sonkki ${ }^{1}$, Sami Myllymäki ${ }^{2}$, Nuutti Tervo ${ }^{1}$, Marko E. Leinonen ${ }^{1}$, Maciej Sobocinski ${ }^{2}$, Giuseppe Destino ${ }^{1}$, \\ Aarno Pärssinen ${ }^{1}$ \\ ${ }^{1}$ Centre for Wireless Communications, University of Oulu, Oulu, Finland, email: marko.sonkki@oulu.fi \\ ${ }^{2}$ Microelectronics Research Unit, University of Oulu, Oulu, Finland, email: sami.myllymaki@oulu.fi
}

\begin{abstract}
The paper presents simulated and measured results of a large millimeter-wave antenna array, designed by keeping mind the particular interests for proof of concepts in 5G demonstrations in South-Korean Winter Olympics 2018. The array consist of $16(2 \times 8)$ unit cells, each having four $(2 \times 2)$ linearly polarized patch elements exited with the same amplitude and phase. The desired $-10 \mathrm{~dB}$ impedance bandwidth for the array is from $25.65 \mathrm{GHz}$ to $27.50 \mathrm{GHz}$, and the proposed structure achieves lower than $-30 \mathrm{~dB}$ mutual coupling between the unit cells. The presented simulation and measurement results show good match with each other, as well as with the specifications. The radiation pattern is measured element by element at $27 \mathrm{GHz}$, and the results are summed in post-processing to perform the array factor. Sidelobe levels are $15 \mathrm{~dB}$ below the maximum gain, whereas the measured maximum gain is around $20 \mathrm{~dB}$ as the numerical results predicted $21.5 \mathrm{~dB}$.
\end{abstract}

Index Terms - 5G antennas, beamforming, beam-steering, massive-MIMO, patch antenna array, phased array, subarray, unit cell.

\section{INTRODUCTION}

One of the key drivers for the development of the next generation of communication system, i.e., the $5 \mathrm{G}$, is the demand of 10-times higher communication data rates. This means up to 2 Gbps peak rate in downlink, and 1 Gbps in uplink compared to the current Long Term Evolution (LTE) systems. A promising and concrete approach is to exploit the large available spectrum available in the Ka-band (26.5-40 GHz) [1] to allow wider transmission bandwidths from $100 \mathrm{MHz}$ up to $1 \mathrm{GHz}$. However, to achieve data rates at Ka-band requires the usage of large antenna arrays with adaptive beamforming capabilities against the high path-loss and sensitivity of the radio-link for dynamic obstructions. In [2], for instance, the results of a channel measurement campaign at $28 \mathrm{GHz}$ shows how line-of-sight (LOS) and multipath components of the radio channel are highly affected by moving objects, e.g. cars.

Millimeter waves (mm-Waves) easily employ large antenna arrays to obtain a sufficient array gain for longer communication coverage, beamforming capabilities for beam adaptation, and MIMO-OFDM (Multiple-Input and Multiple-Output - Orthogonal Frequency Division Multiplexing) with high order modulation schemes to achieve the desired data rate. The mm-Wave wireless backhaul link, which for the proposed antenna array is designed, need to meet the following requirements [3]: 1) long-coverage (a few hundreds of meters), 2), $>2$ Gbps data-rate, 3) capability of MIMO transmissions, and 4) adaptive beamforming. Details of the actual system specifications with implementation architecture, system specifications, and baseband configurations for what the antenna array is designed, can be found in [4][5]. The designed antenna array will be integrated into a radio unit, which includes two identical radio cards with 16 transceivers in each. Thus one radio unit has two antenna arrays which may be in the same or in the opposite polarization depending on MIMO requirements of the radio link. The implemented mobile backhaul uses similar radio units at both ends of the link.

To design a mm-Wave antenna array with large bandwidth and high gain for 5G network, enormous research effort have been proposed to use modified feeding network techniques for patch and new material [6]-[8]. Recently, a comparative review study on $5 \mathrm{G} \mathrm{mm}$-Wave array antenna has been done for outdoor mm-Wave wireless communications, considering the array gain and directivity factors [9]. Radiation pattern of four different antenna array architectures have been investigated including; $8 \times 8$ rectangle array, 64 circular array, 61 hexagonal array and 16 crisscross array.

To enhance radio capacity data rate, by reducing antenna $3 \mathrm{~dB}$ beamwidth from $65^{\circ}$ to $30^{\circ}$, it can increase the capacity of mm-Wave cellular networks operating at $28 \mathrm{GHz}$ frequency roughly by three times at a distance of $220 \mathrm{~m}$ from the base station (BS) [10]. This means the antenna beamwidth have an impact on network performance. Mutual coupling in antenna arrays and its feeding networks have been subjects of intense research for several decades. Several reducing techniques have proposed including suppressing surface waves propagation using electromagnetic band gap (EBG) [11], slitted on the ground plan [12]. Also, a comprehensive review study has been done to study and reduce the mutual coupling impact on planar array antennas performance [13].

This paper is proposing a massive-MIMO antenna array design for Winter Olympics in Korea 2018 for the spectrum between $26.65-27.5 \mathrm{GHz}$ [14]. The band has a particular interests for proof of concepts in $5 \mathrm{G}$ demonstrations. The 


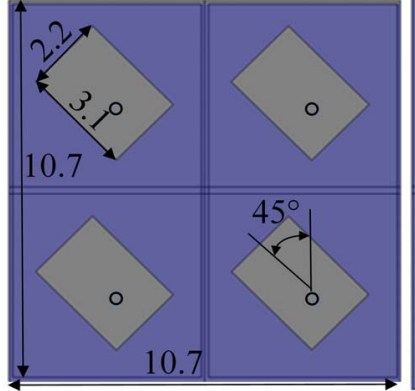

(a)

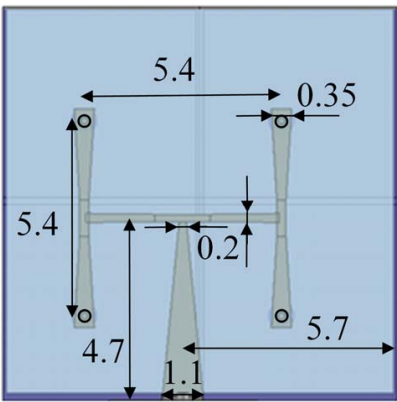

(b)
Figure 1. 2x2 element unit cell present in (a) front with patches and the (b) feed network below ground plane.

array consists of unit cells, which each has $2 \times 2$ patch antennas with feed network, and array structure proposes low mutual coupling between the unit cells. The unit cell is linearly-polarized and each patch element is excited with the same amplitude and equal phase. The total number of patch antennas in the array matrix is $4 \times 16$ elements, meaning $2 \times 8$ unit cells, leading totally to 64 elements.

\section{Structure of Unit CELl AND ANTEnNA ArRay}

This section describes the antenna array designed and developed for massive-MIMO demonstrator. The array consists of $2 \times 2$ patch antenna element unit cells. Each patch element in the unit cell is linearly-polarized and they are excited with the same amplitude and equal phase. Thus, for example phasing between the patch elements within the unit cell is not possible because of the fixed feeding network. The total array matrix configuration is $2 \times 8$ unit cells, leading to 64 patch elements in total. The section also describe the antenna structure with two vertically aligned unit cells as a smallest combination for azimuth plane beamforming as beamforming in elevation is not considered. CST Microwave Studio is used as a simulation tool.

\section{A. $2 \times 2$ Element Unit Cell}

This subsection presents the structure of the $2 \times 2$ element unit cell. The structure and the dimensions are presented in Fig. 1. One unit cell consist of four patches which are oriented in $-45^{\circ}$ polarization against z-axis (see Fig. 6). The size of the unit cell is $10.7 \times 10.7 \mathrm{~mm}^{2}$ and the patch separation is half a wavelength $(=5.4 \mathrm{~mm})$ at $28 \mathrm{GHz}$. Each patch element is fed thought the ground plane with a $200 \mu \mathrm{m}$ diameter via. Moreover, each via is connected to the feed network as presented in Fig. 2. The size of the unit cell was driven by sizes of the available radio components on open markets, such as amplifier.

The individual patch element are designed into a cavity of height of $0.78 \mathrm{~mm}$. This is to improve isolation between the inter-elements in the unit cell but also between the unit cells in the array. A low loss RF laminate, Isola Astra MT77, is used to manufacture the PCB (Printed Circuit Board) structure. The metal cover is placed over the feed network to prevent the backward radiation.

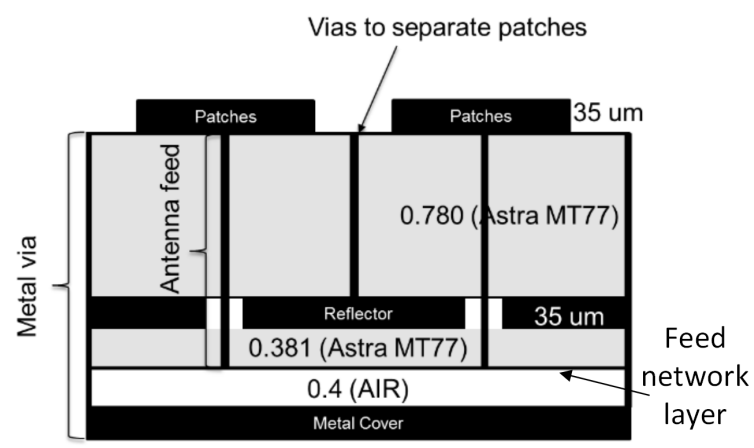

Figure 2. The cross section of the PCB unit cell structure.

\section{B. Two Vertically Aligned 2x2 Element Unit Cells}

This section describes the antenna matrix with two vertically aligned unit cells, presented in Fig. 1. This kind of setup can be seen as a smallest combination of the unit cells in the antenna array as the purpose is not to use beamforming in the elevation plane (Theta in Fig. 6) but only in azimuth plane (Phi in Fig. 6). Also with this arrangement, the mutual coupling between the adjacent unit cells can be studied. The results are presented in the Sections III B with simulated impedance matching, mutual coupling between the unit cells, and the $3 \mathrm{D}$ radiation pattern at $27 \mathrm{GHz}$.

\section{C. $4 x 16$ Element Antenna Array}

Fig. 3 present a photograph of the $2 \times 8$ antenna matrix prototype with 16 unit cells. Thus, the total number of patch elements in the array matrix become 64 and the physical size of the array is $90 \times 34 \mathrm{~mm}^{2}$ (Width $\mathrm{x}$ Height). The PCB structure is presented on both sides without SMPMconnectors and the metal cover over the feed network.

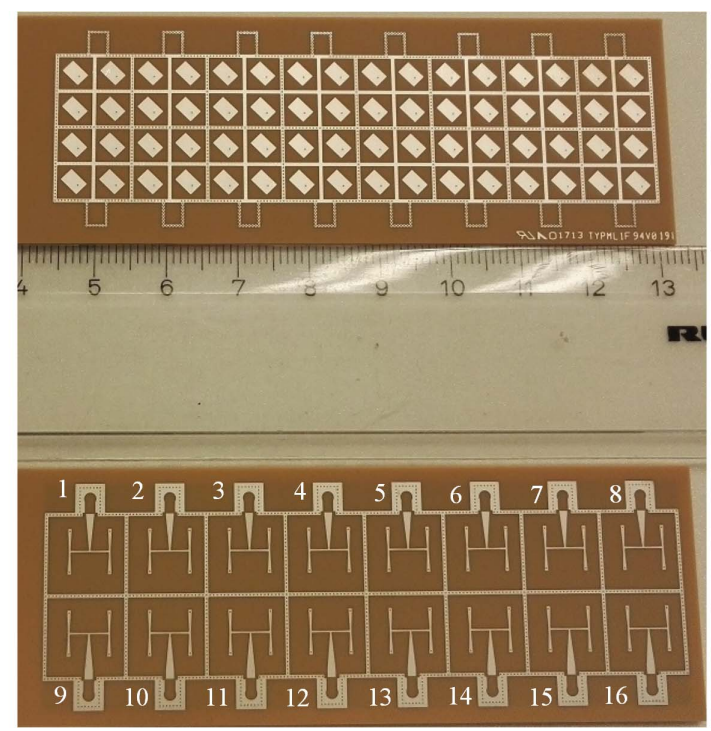

Figure 3. Photograph of the prototype antenna array. Above the antenna array is presented whereas below one can see the feeding network. In the arrays matrix, the antenna ports are marked to the feed network layer from 1 to 8 , from left to right, and the lower line from 9 to 16 . 


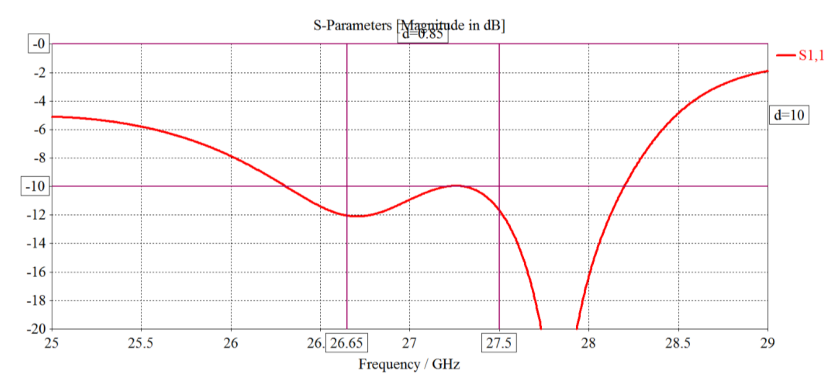

(a)

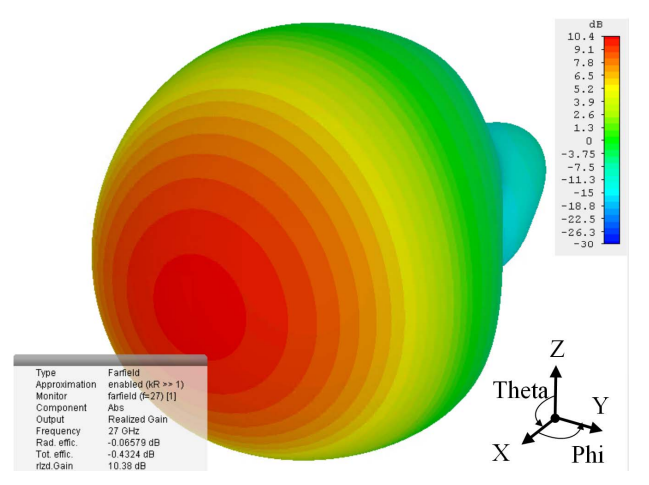

Figure 4. Simulated (a) impedance matching and (b) 3D radiation pattern at $27 \mathrm{GHz}$ of the unit cell presented in Fig. 1. The maximum gain is $10.4 \mathrm{~dB}$ and the total efficiency $-0.43 \mathrm{~dB}$.

\section{RESULTS}

The simulation and measurement results presented in this section are based on the goal of the $-10 \mathrm{~dB}$ impedance bandwidth set to the $26.65-27.50 \mathrm{GHz}$. Section III A presents the simulated results of the unit cells, Section III B describes the simulated results of the two vertically aligned unit cells, and finally Section III C shows the measured results of the full antenna array.

\section{A. $2 x 2$ Element Unit Cells}

Fig. 4 (a) presents the simulated frequency response and $3 \mathrm{D}$ radiation pattern of the unit cell, presented in Fig. 1. As it can be observed, the simulated results show larger bandwidth potential as the required bandwidth, which is $26.65-27.50 \mathrm{GHz}$. At the aforementioned bandwidth, the unit cell shows $-0.43 \mathrm{~dB}$ total efficiency, and the maximum realized gain at $27 \mathrm{GHz}$ is $10.40 \mathrm{dBi}$, as shown in Fig. 4 (b).

\section{B. Two Vertically Aligned $2 \times 2$ Element Unit Cells}

Fig. 5 (a) present the simulated S-parameters of two vertically aligned unit cells. Both unit cells have the same patch orientation and the feed network (Fig. 1) with the cross section profile as presented in Fig. 2. The both unit cells satisfy the $-10 \mathrm{~dB}$ impedance bandwidth and the isolation between the antenna ports is better than $35 \mathrm{~dB}$. Fig. 5 (b) presents the simulated $3 \mathrm{D}$ radiation patterns and as it can be observed, the maximum gain is $13.3 \mathrm{~dB}$, and the total efficiency $-0.37 \mathrm{~dB}$. The increase in gain well corresponds

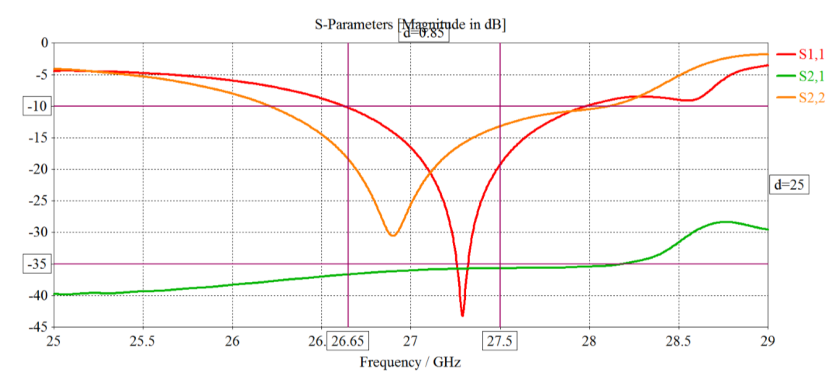

(a)

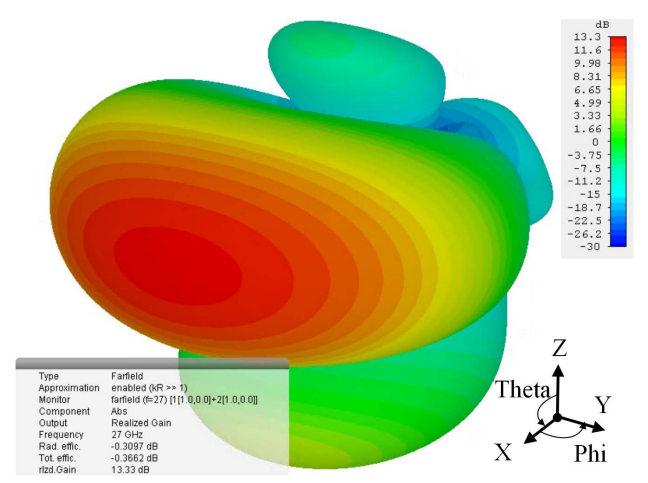

Figure 5. Simulated (a) impedance matching and (b) 3D radiation pattern at $27 \mathrm{GHz}$ of the two vertically orientated (in direction $\mathrm{Z}$ ) unit cells, presented in Fig. 1. The maximum gain is $13.3 \mathrm{~dB}$ and the total efficiency $-0.37 \mathrm{~dB}$.

with the theory of $3 \mathrm{~dB}$ increment when array size is doubled.

\section{C. $4 x 16$ Element Antenna Array}

Fig. 6 presentens the prototype antenna and the setup used to measure the array pattern. The radiation patterns of each unit cells are first measured element by element. The passive unit cells are terminated to $50 \Omega$ standard load. The receiving antenna was linearly polarized standard gain horn antenna. The results are summed to an array factor in postprocessing by combining the measured complex value, adding the free space loss, and to subtracting the standard horn gain at $27 \mathrm{GHz}$.

The measured impedance matching of the 16 ports are presented in the Fig. 7 (a) and (b). As it can be noticed, the results are within the required bandwidth with $<-8 \mathrm{~dB}$ impedance matching. The variation in the results is assumed to become from the manufacturing tolerances as simulations were predicting that only $50 \mu \mathrm{m}$ changes in the structure has significant effect to the antenna performance in terms of impedance matching.

In Fig. 7 (c), the measured mutual coupling between the selected ports are presented. The presented mutual couplings are chosen with some of the adjacent ports and with a nearby ports as the measured matrix become very large (16x16). From the measured results it can be observed that the mutual coupling is smaller than $-30 \mathrm{~dB}$. As a conclusion, when comparing the results in Fig. 4 and 5, the simulations are well correlating with the measured ones. 


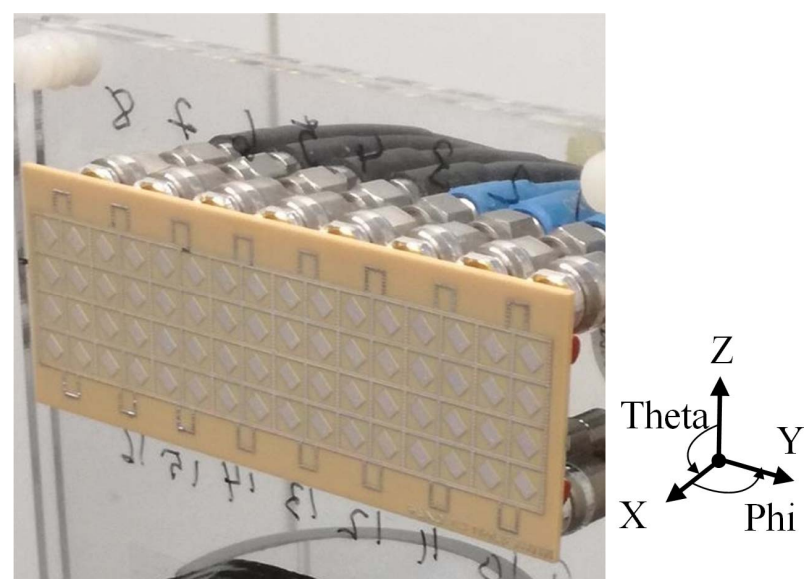

Figure 6. Photograph of the prototype antenna array in radiation pattern measurements.

Fig. 7 (d) presents the comparison of simulated measured radiation patterns in azimuth plane (XY-cut) at $27 \mathrm{GHz}$. As it can be observed, the sidelobe level is $15 \mathrm{~dB}$ below the maximum gain which is predicted by simulations. The maximum gain is around $20 \mathrm{~dB}$ as the numerical results predicts $21.5 \mathrm{~dB}$. The overall $1.5-2 \mathrm{~dB}$ difference in the gain comes from the adaptor losses. Thus, it can be concluded that there is a good correlation between the simulated and measured results.

\section{CONCLUSIONS AND FUTURE WORK}

The paper presented antenna array structure which consists of $16(2 \times 8)$ unit cells, each having $4(2 \times 2)$ patch elements. The unit cell is linearly-polarized and each patch element is excited with the same amplitude and equal phase. The total number of patch antennas in the array matrix is 64 $(4 \times 16)$ elements. The presented simulation and measurement showed $<-8 \mathrm{~dB}$ impedance matching over $26.65-27.50 \mathrm{GHz}$ bandwidth. The radiation patterns were measured element by element and the results are summed in post-processing. Sidelobe levels are $15 \mathrm{~dB}$ below the maximum gain, and the maximum gain is around $20 \mathrm{dBi}$ as the numerical results predicted $21.5 \mathrm{dBi}$. In general, a good correlation between the simulated and measured results can be concluded.

As a future work, the antenna array will be implemented for the final backhaul link, and bandwidth will be enlarged to the bandwidth from $24.25 \mathrm{GHz}$ to $29.20 \mathrm{GHz}$, including both $\pm 45^{\circ}$ polarization components. One antenna array will be connected to a 16 port transceiver, designed in the 5 G CHAMPION project. With such an RF phased array transceiver, an active measurements will be conducted over-the-air (OTA).

\section{ACKNOWLEDGMENT}

The research leading to these results has received by funding of the European Union H2020 5GPPP under grant n. 723247, supported by the Institute for Information \& communications Technology Promotion (IITP), grant funded by the Korean government (MSIP) (No.B0115-16-0001, 5G CHAMPION), by Finnish Funding Agency for

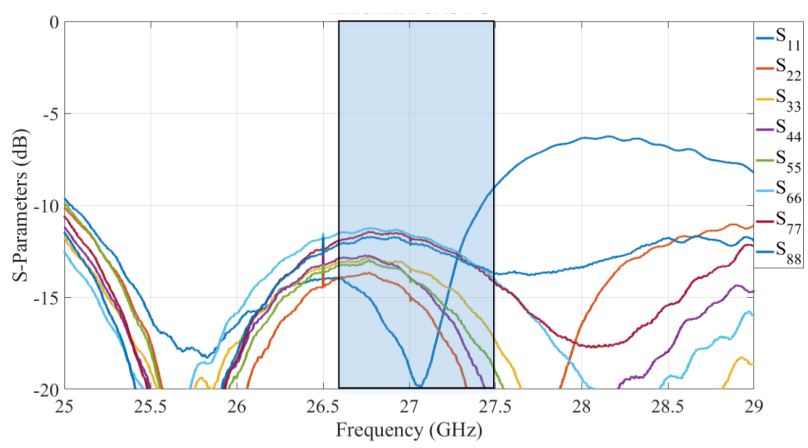

(a)

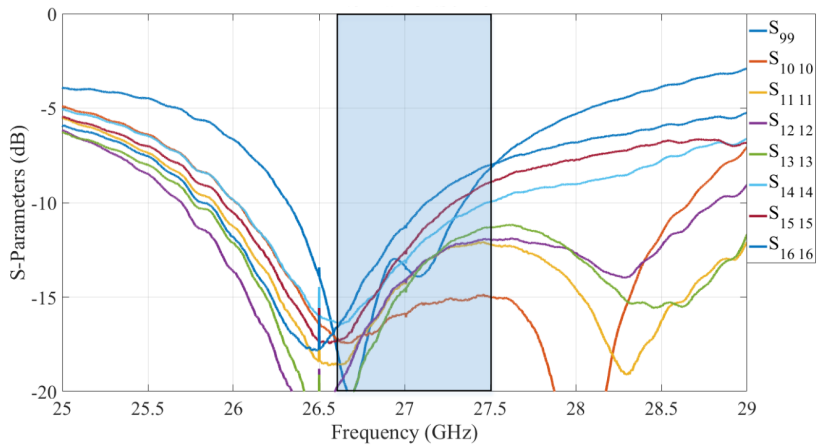

(b)

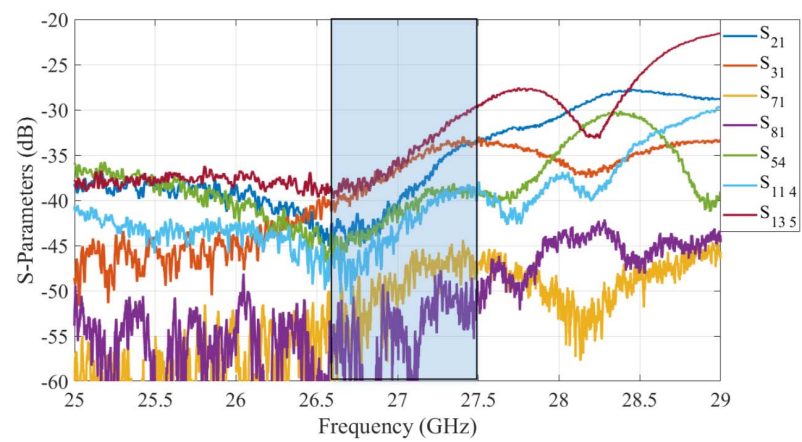

(c)

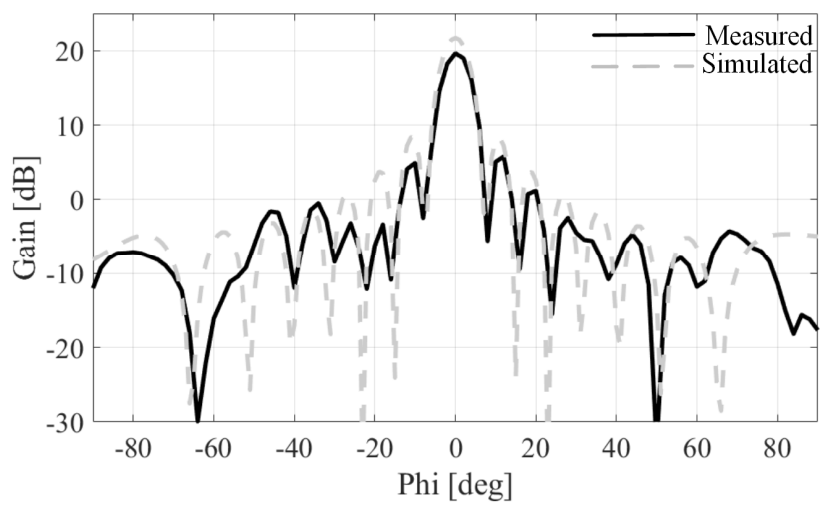

(d)

Figure 7. Measured results with blue box representing required 26.65-27.5 GHz bandwidth (a) Ports 1-8, (b) Ports 9-16, (c) mutual coupling between selected antenna ports, and (d) comparison of simulated and measured radiation patterns at $27 \mathrm{GHz}$ in azimuth plane (XY-cut). 
Innovation, and by Nokia Networks Oulu and Finnish Funding Agency for Innovation under project 5G FOAMED.

The authors are also very grateful to Mr. Juho Rivinoja, Mr. Irfan Muhammad, and Mr. Nédio C. da S. Neddef who performed the RF measurements of the study.

\section{REFERENCES}

[1] F. Boccardi et al., "Five disruptive technology directions for 5G," IEEE Communications Magazine, vol. 52, no. 2, Feb. 2014, pp. 7480 .

[2] R. J. Weiler et al., "Environment Induced Shadowing of Urban Millimeter-Wave Access Links," IEEE Wireless Communications Letters, vol. 5, no. 4, Aug. 2016, pp. 440-443.

[3] G. Destino et al., "Deliverable D2.2 5GCHAMPION Key Performance Indicator and use-cases defined and specification document written", 5GChampion project public document, 2017.

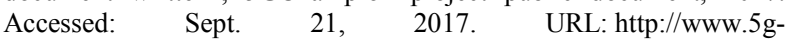
champion.eu/Documents/5GCHAMPION_D2.2_Final.pdf

[4] E. C. Strinati et al., "Deliverable D1.2 First period report", 5GChampion project public document, 2017. Accessed: Sept. 21, 2017.

URL: http://www.5g-champion.eu/Documents/5GCHAMPION_D1.2 _Final.pdf

[5] W. Tavernier et al., "Deliverable D2.1 5G CHAMPION architecture, API- and interface document", 5GChampion project public document, 2017. Accessed: Sept 21, 2017. URL: http://www.5gchampion.eu/Documents/5GCHAMPION_D2.1_Final.pdf

[6] H. Aliakbari, A. Abdipour, R. Mirzavand, A. Costanzo, and P. Mousavi, "A single feed dual-band circularly polarized millimeterwave antenna for 5G communication," in 2016 10th European Conference on Antennas and Propagation (EuCAP), 2016, pp. 1-5.

[7] M. T. Passia, M. Nitas, and T. V. Yioultsis, "A fully planar antenna for millimeter-wave and 5G communications based on a new CSRRenhanced substrate-integrated waveguide," in 2017 International Workshop on Antenna Technology: Small Antennas, Innovative Structures, and Applications (iWAT), 2017, pp. 183-186.

[8] S. F. Jilani and A. Alomainy, "Planar millimeter-wave antenna on low-cost flexible PET substrate for 5G applications," in Antennas and Propagation (EuCAP), 2016 10th European Conference on, 2016, pp. 1-3: IEEE.

[9] J. Zhang, X. Ge, Q. Li, M. Guizani, and Y. Zhang, "5G MillimeterWave Antenna Array: Design and Challenges," IEEE Wireless Communications, vol. 24, no. 2, pp. 106-112, 2017.

[10] A. T. Nassar, A. I. Sulyman, and A. Alsanie, "Radio capacity estimation for millimeter wave $5 \mathrm{G}$ cellular networks using narrow beamwidth antennas at the base stations," International Journal of Antennas and Propagation, vol. 2015, 2015.

[11] E. Rajo-Iglesias, Q.-T. Ó, and L. Inclan-Sanchez, "Mutual Coupling Reduction in Patch Antenna Arrays by Using a Planar EBG Structure and a Multilayer Dielectric Substrate," IEEE Transactions on Antennas and Propagation, vol. 56, no. 6, pp. 1648-1655, 2008.

[12] C.-Y. Chiu, C.-H. Cheng, R. D. Murch, and C. R. Rowell, "Reduction of mutual coupling between closely-packed antenna elements," IEEE Transactions on Antennas and Propagation, vol. 55, no. 6, pp. 17321738, 2007.

[13] H. Chen, X.-F. Ji, L.-J. Jiang, and Y.-X. Zhang, "Design and implementation of an X-band pulsed solid-state power amplifier with high power and high efficiency using radial waveguide combiner," Progress In Electromagnetics Research C, vol. 21, pp. 113-127, 2011.

[14] M. Mueck et al., "5G CHAMPION - Rolling out 5G in 2018," IEEE Globecom Workshops, Dec. 2016, pp. 1-6. 